\title{
Mind your state: Insights into antidepressant nonadherence
}

\author{
De Wet Wolmarans' ${ }^{1}$, Sarel J. Brand ${ }^{1}$ \\ 'Centre of Excellence for Pharmaceutical Sciences, Division of Pharmacology, Faculty of Health Sciences, \\ North-West University, Potchefstroom \\ Corresponding author, email: dewet.wolmarans@nwu.ac.za
}

\begin{abstract}
Major depressive disorder (MDD) is an insidious disease and affects up to $15 \%$ of the global population. Although MDD responds to a wide range of pharmacological treatment options, a number of factors, i.e. not adhering to treatment for at least 4-12 months, contribute to antidepressants not being highly effective. In an attempt to aid clinicians in improving the adherence rates among MDD patients, the current paper will divulge in more detail the possible explanations of why individuals with MDD find it difficult to adhere to prescribed regimens.
\end{abstract}

\section{Introduction}

Major depressive disorder (MDD) is a severe and debilitating condition ${ }^{1}$ that occurs in $8-15 \%$ of the global population ${ }^{2}$ while locally one out of every ten South Africans suffer from depressive episodes at some point in their lifetime. ${ }^{3}$ Moreover, almost 50\% of patients diagnosed with MDD suffer from co-morbid anxiety disorders, e.g. panic disorder and generalised anxiety disorder (GAD). ${ }^{1}$ As such, the diagnosis of MDD is complex, involving a systematic process that must consider and evaluate a range of core symptoms. ${ }^{4}$ Although MDD is a chronic psychiatric disorder, most patients only seek medical care during acute depressive episodes of which the majority of cases are reported in general, and not psychiatric practice. ${ }^{5}$ Although the acute manifestation of symptoms typically prompts patients to consult medical professionals, the pharmacological treatment of MDD follows an intensive and chronic course that requires uninterrupted adherence for at least 4-12 months. ${ }^{6}$ This being said, it has been demonstrated that up to $80 \%$ of patients are noncompliant by either missing daily doses or withdrawing therapy altogether. ${ }^{7-9}$ As such, in an attempt to support healthcare providers in addressing the challenges met when patients diagnosed with MDD fail to comply with treatment, the current paper aims to broaden our understanding of the clinical, social and economic consequences of, and reasons for, antidepressant nonadherence.

\section{Getting the facts straight}

- Globally, an estimated 16 million annual attempts at suicide result in nearly 800000 deaths. ${ }^{10}$

- 11 million of these attempts are preceded by depressive episodes. ${ }^{11}$

- Patients diagnosed with MDD have an estimated 5-10 years decrease in life expectancy ${ }^{12}$ not only related to suicidal behaviour, but mostly due to comorbid cardio-metabolic disorders. ${ }^{13}$

- Only $10 \%$ of MDD patients receive adequate treatment. ${ }^{9}$

- Even with intensive pharmacological treatment, only $60-70 \%$ of patients achieve remission. ${ }^{14}$

\section{The importance of antidepressant adherence}

\section{While drugs may be slow to act, humans are slower at learning}

Although increasing evidence demonstrates MDD to be associated with disorders that adversely affect normal sleep architecture, the immune system and metabolic function ${ }^{15}$, most of the currently available antidepressants act by targeting monoamine transmission in the brain. However, due to its dependence on a gradual change in central monoamine function ${ }^{15}$ and cognitive neurocircuitry, antidepressants have a slow onset of action. On the other hand, novel evidence indicates that long-term emotional learning processes may play a key role in antidepressant action whereby patients need to re-establish the emotional meanings of previously negative response-outcome contingencies while being under the influence of antidepressant action. ${ }^{16}$ This highlights the potential of psychotherapy as an important add-on to drug-based treatment approaches.

Clinical example:Tom (pseudonym) was consulted at your practice in 2014 following a major depressive episode and was diagnosed with MDD. One of the most prominent factors that triggered his depression was conversations with his ex-wife that concerned their children. Although being in no position to change his reality, confrontations with his ex-wife constantly caused him to doubt his adequacy as a father. However, being a sufferer from $M D D$, he was unable to realise that during the dialogues with his wife, neither he, nor his suitability as a father was the subject 
of discussion, but that the best interests of their children were at stake. While being treated for MDD, Tom gradually learned that although the subject of these conversations fundamentally remained the same and that they would always remind him of his life before the divorce, they had no emotional meaning other than providing a stable arrangement for their children.

\section{What is remission worth to you?}

It has repeatedly been demonstrated that nonadherence to antidepressant treatment results in poor clinical ${ }^{17}$, social ${ }^{18}$ and economic $^{19}$ outcomes. As such, patients not adhering to at least four to twelve months of uninterrupted antidepressant use are at an increased risk for symptom relapse, treatment resistance and hospitalisation. ${ }^{72} 20$ Over time this translates to higher rates of unemployment, marital discord and unstable social relationships. ${ }^{21}$ Furthermore, although adherent patients incur significantly higher drug costs compared to noncomplying individual $\mathrm{s}^{22}$, their total expenditure based on all depressionrelated medical interventions is often much lower.

\section{Why is treatment adherence so difficult?}

\section{The personal character of a depressed mood}

Considering that depression is at its core a complex emotional condition that is not easily defined and subject to personal bias, various studies have aimed to elucidate the role of patient experience in the treatment of MDD. ${ }^{23}$ Indeed, individuals that present with symptoms of MDD are often unconvinced that the feelings they experience, i.e. anhedonia, carelessness or fatigue, may be traits of a larger psycho-biological disorder that necessitates pharmacological intervention. ${ }^{24}$ As such, patients will often avoid seeking medical attention, and they regularly find it difficult to commit to treatment once they do consult a healthcare professional. A number of social and personal factors may contribute to patients not choosing to accept the reality of their mood state and being hesitant to disclose their emotions. Hence, feelings of vulnerability to interpersonal scrutiny and stigmatisation, thinking that partners or next of kin will set unrealistic goals for treatment, and knowing themselves better than anyone else often lead these individuals to believe that they are able to self-regulate their behaviour. ${ }^{23}$

\section{Trust is hard to earn}

The concept of trust is perhaps the most important factor determining whether patients will adhere to treatment. ${ }^{25}$ With respect to MDD, trust can broadly be conceptualised on two levels, i.e. 1) accepting the neurobiological nature of the condition and believing that adhering to treatment will result in symptom attenuation; and 2) trusting healthcare practitioners to manage the clinical prognosis of the condition. Indeed, the slow onset of action of antidepressants and sometimes severe nature of their side-effects as well as the fact that treatment adherence is not a guarantee to achieve symptom remission lead to $60-80 \%$ of patients being hesitant about the likelihood of a positive outcome with continuous drug treatment. ${ }^{23,26}$ Moreover, recent studies highlighted the impact of distrustful relationships between MDD patients and their clinicians in treatment nonadherence. ${ }^{24,25}$ Particularly during phases of nonadherence, patients have reported that they deliberately avoid therapists for three main reasons, i.e. 1) they do not believe that their clinicians are competent to appreciate their own perceived need for change; 2 ) the prescribing clinician will often argue for continuing or augmenting the current treatment regimen; and 3) they experience consultations with their clinicians as taxing intellectual debates concerning the necessity to make changes to current, and in their view, failing interventions. ${ }^{23}$ Interestingly, these feelings are exacerbated by the differing perceptions of psychiatrists and general practitioners regarding MDD. ${ }^{5}$ Also, as patients often switch between clinicians, inter-professional differences in opinion and prescribing patterns ${ }^{27}$ contribute to patients not trusting their therapists with managing their disease. ${ }^{5,23}$

\section{Fostering patient patients: Intervening on their behalf}

Taken from the above, clinicians can intervene on various levels to improve antidepressant adherence:

- Accept that MDD is intrinsically founded in both the psychological and biological domains. As such, it will be difficult to convince patients that they will be cured simply by prescribing antidepressants.

- Personal bias and self-regulation will more often than not be part of discussions between clinician and patient. Essentially, it is not a case of your patients trying to know better, but rather informing you about how they feel. In fact, pertaining to mood disorders, conversation is the only means of diagnosis and clinical follow-up.

- It's all about trust. From the first consultation, be honest about the complexity of MDD and the uncertainty of treatment outcome. Schedule longer first appointments that allow time for careful diagnosis and accept that once you initiate treatment, a commitment to follow up must be made.

- Importantly, rather refer than desert. Treating patients with psychiatric illness, especially conditions that may trigger suicidal behaviour, is different from alleviating pain or curing infection. It requires time, an ability to listen and the assurance of being there.

\section{Conclusion}

MDD is a highly heterogeneous and complex psycho-biological mood disorder that necessitates chronic, uninterrupted pharmacological treatment. However, a number of factors contribute to the fact that up to $80 \%$ of patients diagnosed with MDD do not adhere to drug treatment. Key to this is the relative uncertainty of treatment outcome and a lack of trust between patients and their therapists. While patients have a responsibility to commit to therapist-initiated interventions, clinicians are challenged to change their perceptions of MDD and to accept that treating mood disorders involves a unique and singular approach. 


\section{References}

1. Kessler RC, et al. Lifetime prevalence and age-of-onset distributions of mental disorders in the World Health Organization's world mental health survey initiative. World Psychiatry, 2007;6(3):168-176.

2. Andrade $L$, et al. The epidemiology of major depressive episodes: Results from the International Consortium of Psychiatric Epidemiology (ICPE) Surveys. International Journal of Methods in Psychiatric Research, 2003;12(1):3-21.

3. Tomlinson $M$, et al. The epidemiology of major depression in South Africa: Results from the South African Stress and Health study. South African Medical Journal, 2009;99(5):368-373.

4. American Psychiatric Association. Diagnostic and statistical manual of mental disorders. Vol. 5. 2013, Washington, DC: American Psychiatric Association.

5. Davidsen AS, Fosgerau CF. What is depression? Psychiatrists' and GPs' experiences of diagnosis and the diagnostic process. International Journal of Qualitative Studies on Health and Well-being, 2014;9.

6. Geddes JR, et al. Relapse prevention with antidepressant drug treatment in depressive disorders: A systematic review. Lancet, 2003;361(9358):653-661.

7. Ho SC, et al. Clinical and economic impact of non-adherence to antidepressants in major depressive disorder: A systematic review. Journal of Affective Disorders, 2016;193:1-10.

8. Keller MB, et al. Optimizing outcomes in depression: Focus on antidepressant compliance. International Clinical Psychopharmacology, 200217(6):265-271.

9. Epstein I, Szpindel I, Katzman MA. Pharmacological approaches to manage persistent symptoms of major depressive disorder: rationale and therapeutic strategies. Psychiatry Research, 2014;220(S1);S15-S33.

10. World Health Organization (WHO), Preventing suicide: A global imperative. 2014;92.

11. Trivedi MH, Daly EJ. Treatment strategies to improve and sustain remission in major depressive disorder. Dialogues in Clinical Neuroscience, 2008;10(4):377-384.

12. Ciechanowski PS, Katon WJ, Russo JE. Depression and diabetes: Impact of depressive symptoms on adherence, function, and costs. Archives of Internal Medicine, 2000; 160(21):3278-3285.

13. Mclntyre RS, et al. The effect of antidepressants on glucose homeostatis and insulin sensitivity: Synthesis and mechanisms. Expert Opinion on Drug Safety, 2006;5(1):157-168
14. Gaynes BN, et al. What did STAR*D teach us? Results from a large-scale, practical, clinical trial for patients with depression. Psychiatric Services, 2009;60(11):1439-1445.

15. Willner $P$, Scheel-Krüger J, Belzung $C$. The neurobiology of depression and antidepressant action. Neuroscience and Biobehavioral Reviews, 2013;37(10):2331-2371.

16. Roiser JP, Elliott R, Sahakian BJ. Cognitive mechanisms of treatment in depression. Neuropsychopharmacology, 2012;37(1):117-136.

17. Alekhya $P$, et al. Adherence to antidepressant therapy: Sociodemographic factor wise distribution. International Journal of Pharmaceutical and Clinical Research, 2015;7(3):180-184.

18. Kessler RC, et al. Social consequences of psychiatric disorders, II: Teenage parenthood. American Journal of Psychiatry, 1997;154(10):1405-1411.

19. Osterberg L, Blaschke T. Adherence to medication. New England Journal of Medicine, 2005;353(5):487-497.

20. Liu X, Tepper PG, Able SL. Adherence and persistence with duloxetine and hospital utilization in patients with major depressive disorder. International Clinical Psychopharmacology, 2011;26(3):173-180.

21. Kessler RC, et al. Social consequences of psychiatric disorders, I: Educational attainment. American Journal of Psychiatry, 1995;152(7):1026-1032.

22. White TJ, et al. Economic Impact of Patient Adherence with Antidepressant Therapy Within a Managed Care Organization. Disease Management and Health Outcomes, 2003;11(12):817-822.

23. Buus N. Adherence to anti-depressant medication: A medicine-taking career. Social Science and Medicine, 2014;123:105-113.

24. McMullen LM, Herman J. Women's accounts of their decision to quit taking antidepressants. Qualitative Health Research, 2009;19(11):1569-1579.

25. Anderson C, Roy T. Patient experiences of taking antidepressants for depression: A secondary qualitative analysis. Research in Social and Administrative Pharmacy, 2013; 9(6):884-902.

26. Van Geffen ECG, et al. The decision to continue or discontinue treatment Experiences and beliefs of users of selective serotonin-reuptake inhibitors in the initial months-A qualitative study. Research in Social and Administrative Pharmacy, 2011;7(2):134-150.

27. Davies NM, et al. Physicians' prescribing preferences were a potential instrument for patients' actual prescriptions of antidepressants. Journal of Clinical Epidemiology, 2013;66(12):1386-1396. 